\title{
Development of a Multiplex Glycan Microarray Assay and Comparative Analysis of Human Serum Anti-Glycan IgA, IgG, and IgM Repertoires
}

\author{
Sarah V. Durbin, W. Shea Wright, and Jeffrey C. Gildersleeve*(i) \\ Chemical Biology Laboratory, Center for Cancer Research, National Cancer Institute, Frederick, Maryland 21702, United States
}

Supporting Information

\begin{abstract}
Serum antibodies that recognize carbohydrate antigens play a fundamental role in immune defense, homeostasis, and autoimmunity. In addition, they serve as potential biomarkers for a variety of medical applications. For most anti-glycan antibodies found in human serum, however, the origins, regulation, and biological significance are not well understood. Antibody subpopulations that are relevant to a particular biological process or disease are often difficult to identify from the myriad of anti-glycan antibodies present in human serum. While prior studies have examined anti-glycan

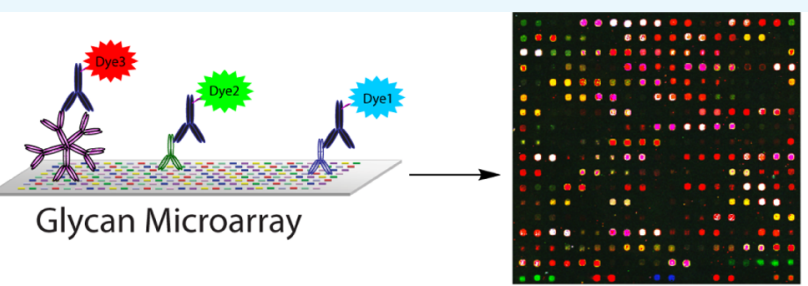

Multiplex assay detects $\lg A$, $\lg G$, and $\lg M$ IgG and/or IgM repertoires, little is known about IgA repertoires or how IgA, IgG, and IgM are related. In this study, we describe the development of a multiplex assay to simultaneously detect IgA, IgG, and IgM on a glycan microarray and its application to studying anti-glycan repertoires in healthy subjects. The multiplex glycan microarray assay revealed unique insights and systems-level relationships that would be difficult to uncover using traditional approaches. In particular, we found that anti-glycan $\operatorname{IgA}, \operatorname{IgG}$, and IgM expression levels appear to be tightly regulated, coordinated within individuals, and stable over time. Additionally, our results help define natural fluctuations over time, which is critical for identifying changes that are beyond normal biological variation.
\end{abstract}

\section{INTRODUCTION}

Human serum contains a diverse collection of antibodies that play important roles in human health and yield valuable insights into immune function. Antibody profiling helps scientists understand how the immune system responds to infections or diseases and identify antibody subpopulations that can be used for diagnosing diseases or managing treatments. Additionally, antibody profiling can be useful for the design and development of vaccines by identifying new target antigens and determining what are the favorable or detrimental immune responses to specific treatment.

Serum contains numerous antibodies that recognize carbohydrate antigens, but this subset has been largely under studied relative to antibodies that bind peptides and proteins. ${ }^{1-3}$ The dearth of knowledge is not due to a lack of importance. Carbohydrate-binding antibodies are involved in many immunological processes, such as tumor surveillance, autoimmunity, allergy, and response to vaccines, and they are useful for clinical diagnoses and detection. For example, anti-glycan antibodies to blood group A and B antigens are relevant for matching donors and recipients in blood transfusions and organ transplants. Rather, the deficiency of information is due to the challenges of profiling of anti-glycan antibodies, such as the lack of access to glycans and poor throughput of traditional assays.

Glycan microarrays provide a powerful, high-throughput tool to profile serum antibody populations and identify specific subsets with clinical or biological relevance. Many different carbohydrates are immobilized on glass slides in a spatially defined arrangement, allowing one to profile serum antibody levels for hundreds of glycans in a single experiment while only using minimal amounts of clinical samples and scarce carbohydrates. Glycan microarrays have been used in numerous studies to profile glycan-binding serum antibodies (for some recent examples, see refs; ${ }^{4-16}$ for a recent review, see ref 1 ). These studies have primarily focused on either total antibody levels or IgG levels to various glycans. IgM has been evaluated in some studies, but very few studies have included IgA antibodies. In most cases, researchers only evaluated a single antibody type per experiment. Because each antibody type uses one array per serum sample, the additional arrays required to evaluate multiple antibody isotypes or subclasses becomes prohibitive. For example, in a recent study, we analyzed serum antibodies in about 240 serum samples, with each being tested in duplicate. ${ }^{16}$ To measure one additional isotype or subclass, nearly 500 more array experiments would have been required. For these reasons, little is known about anti-glycan IgA repertoires or relationships between anti-glycan IgA, IgG, and IgM antibodies. Furthermore, researchers may be missing important responses from isotypes that are not being evaluated.

Received: August 31, 2018

Accepted: November 27, 2018

Published: December 7, 2018 
Multiplex assays enable parallel analysis of multiple analytes in a single experiment and provide numerous advantages. The ability to profile multiple antibody isotypes in a single glycan microarray experiment would provide many advantages over existing assays. In addition to various practical benefits, such as generating more information from fewer arrays and less reagents, a multiplex assay provides identical assay conditions for each isotype leading to reduced variability relative to measuring each in separate experiments. Simultaneous measurements of IgA, IgG, and IgM would also improve our understanding of relationships between isotypes and whether variations in anti-glycan IgA, IgG, and IgM are coordinated or independent of each another. Lastly, the increase in data would facilitate a better understanding of anti-glycan immunity to infection, disease, and vaccines and a more rapid identification of biomarkers.

Simultaneous detection of two isotypes or analytes on a microarray is well established. Multiplex assays for measuring three or more isotypes/analytes on a microarray have been reported recently for protein microarrays, ${ }^{17-19}$ but no such assays have been developed for measuring IgA, IgG, and IgM using a glycan microarray. At the outset, it was not clear if the same multiplex strategy would be suitable for glycan microarrays, as serum anti-glycan antibodies have significant differences in affinity, selectivity, and isotype usage relative to antibodies that bind proteins. In addition, the reported multiplex assays for protein arrays have some drawbacks. In prior studies of multiplex assays on protein microarrays, fluorescence signals from the dyes were detected in multiple laser channels, leading to differences in signals for the multiplex versus singleplex assays. To account for this bleed-through or "spillover" effect, one assay used narrow band filters, resulting in lower sensitivity, and another assay used multiple correction factors to adjust the signals, which increases variability. Second, the studies provided limited validation of the multiplex assays (i.e., testing with a single sample).

In this study, we describe the development and application of a multiplex assay to simultaneously detect IgA, IgG, and IgM on a glycan microarray. The development of the multiplex assay proved more challenging than expected, and below we describe various issues that arose and how we addressed those problems. Once the multiplex assay was optimized, it was used to study $\operatorname{IgA}$ IgG, and IgM repertoires in healthy subjects over 4-12 weeks. The study enabled new insights into relationships among anti-glycan $\operatorname{IgA}, \operatorname{IgG}$, and $\operatorname{IgM}$ as well as a better understanding of natural biological variations of anti-glycan antibodies over time within humans.

\section{RESULTS}

Initial Planning. The first goal of this study was to design and validate a multiplex assay to simultaneously profile three antibody isotypes in human serum. The main criterion for a multiplex assay is that the signals measured in the multiplex system are equivalent to the signals measured for each individual assay. On the basis of the published reports of bleed-through for multiplex microarray assays, we selected our fluorophores and secondary reagents to minimize this problem. Our microarray scanner has three lasers and corresponding filters: a $488 \mathrm{~nm}$ laser with a $520 / 5 \mathrm{~nm}$ filter, a $532 \mathrm{~nm}$ laser with a $572 / 34 \mathrm{~nm}$ filter, and a $635 \mathrm{~nm}$ laser with a $675 / 37 \mathrm{~nm}$ filter. We anticipated that the largest amount of bleed-through would come from the 488 $\mathrm{nm}$ dye being detected by the $532 \mathrm{~nm}$ laser. To minimize this effect, we matched the $488 \mathrm{~nm}$ dye with the secondary reagent that would detect the fewest and weakest signals, anti-IgA. IgM, which typically has the strongest anti-glycan antibody signals in serum, would be detected by the $635 \mathrm{~nm}$ laser. The $532 \mathrm{~nm}$ channel would then be used to measure IgG signals.

Initial Results and IgA Signal Loss. We initially tested a pooled human serum sample (termed reference serum) using the following commercially conjugated secondary antibodies: Alexa Fluor F488 anti-human IgA, DyLight549 anti-human IgG, and Alexa Fluor 647 anti-human IgM. Each isotype was run alone and in combination with the other secondary antibodies to evaluate signal continuity between single and multiplex wells. There were no measurable cross-over of IgG signals into the 635 $\mathrm{nm}$ channel, but some bleed-through of Alexa Fluor 488-labeled IgA into the $532 \mathrm{~nm}$ channel was detected. Unfortunately, we ran into an unexpected and more worrisome problem than the bleed-through-we observed a significant reduction of IgA signal between the single and multiplex wells for many array glycans. For example, the signals for rhamnose and blood group B tetrasaccharide, which usually have some of the largest IgA signals, were 128109 and 98197 RFUs alone but were reduced to 40932 (68\% signal loss) and 55765 (44\% loss), respectively, in the multiplex. The average IgA signal loss was $40 \%$, with 54 array components losing more than $50 \%$ of their singleplex IgA signal and 5 losing more than $75 \%$ of their original IgA signal (see Supporting Information, Figure S2). The presence of 54 array components with decreases of $50 \%$ or more was well beyond what one might expect from experimental variability and particularly concerning as it would cause us to underestimate the IgA serum antibodies and possibly miss significant changes. Signal loss was most prevalent for array components with high IgA and IgG signals.

We considered several possible explanations for the reductions in $\operatorname{IgA}$ signals. First, serum $\operatorname{IgA}, \operatorname{IgG}$, and $\operatorname{IgM}$ molecules can all bind simultaneously to a given array component. Therefore, the various secondary reagents might compete for access to antibodies bound to a given spot. If space is limited, the presence of anti-IgM and/or anti-IgG secondary antibodies could potentially block access of anti-IgA antibodies via steric hindrance. Alternatively, fluorophores on the various secondary antibodies might interact, possibly leading to quenching of fluorescence. For quenching to occur, the dyes would need to have some spectral overlap (which is common between fluorophores excited with 488 and $532 \mathrm{~nm}$ lasers) and be close enough on the array surface for such energy transfer to occur. To distinguish these possibilities, we switched the dyes attached to the anti-IgA and anti-IgM secondary antibodies. In particular, we used Alexa Fluor 488-labeled anti-IgM and Alexa Fluor 647-labeled anti-IgA. When we switched the fluorophores, we found the signal loss to be specific to the $488 \mathrm{~nm}$ fluorophore, supporting the quenching hypothesis rather than steric hindrance.

Strategies To Minimize Dye-Dye Interactions. One approach for minimizing dye-dye interaction was to vary the spectral properties of the dyes to minimize spectral overlap. We tested several other dyes, but no improvements were obtained. An alternative strategy is to reduce the number of fluorophores on each secondary antibody. In principle, lower dye loading would decrease the likelihood that two dyes would be in close enough proximity on the array surface to interact. The commercially available Alexa Fluor 488 anti-IgA reagent had 6.9 fluorophores/antibody, and the commercially available DyLight549 anti-IgG had 5 fluorophores/antibody. We performed conjugation reactions to get ratios of 1.0 Alexa 
Fluor 488 per anti-IgA and 1.5 Alexa Fluor 546 per anti-IgG; we also made a 4.9 Alexa Fluor 546 anti-IgG secondary antibody to use as a reference point, as there is no commercially available secondary antibody with this dye. The lower loaded dyes were then initially tested in a duplex assay detecting $\operatorname{IgA}$ and $\operatorname{IgG}$ antibodies on the array and directly compared to the commercially available dye-labeled secondary reagents. Decreasing the Alexa Fluor 546 loading on the anti-IgG from 4.9 to 1.5 produced about a $50 \%$ improvement (a $50 \%$ decrease in the number of array components with signal loss greater than 50\%). Decreasing the Alexa Fluor 488 loading on the anti-IgA reagent from 6.9 to 1.0 also provided about a $50 \%$ improvement. The combination of low-loaded anti-IgG and low-loaded anti-IgA appeared to eliminate IgA signal loss, at least in this duplex assay. We also note that bleed-through with low-loaded dyes was negligible (see Supporting Information for more details).

Multiplex Assay Validation. The goal of the following experiments was to verify that our selected secondary reagents for the multiplex platform would provide reliable data. We first tested the standard reference serum at a 1:50 dilution using all three low dye-loaded secondary reagents: Alexa Fluor 488 antihuman IgA (1.0 dye/antibody loading), Alexa Fluor 546 antihuman IgG (1.5 dye/antibody loading), and CF633 anti-human IgM (2.2 dye/antibody loading). Each experiment contained duplicate wells of each secondary antibody alone and then all three together. With the low-loaded secondary antibodies, only 8 components had IgA decreases of $50 \%$ or greater. Signal variations of $>50 \%$ for 8 array components out of 500 total array components is within expectations based on normal experimental variation for our microarray data. ${ }^{20}$ When we graphed the singleplex IgA signals versus the corresponding multiplex signals for our low conjugated secondary antibodies, we observed a Pearson correlation constant $(R)$ of 0.98 and a slope of 0.99 indicating highly consistent results (see Supporting Information, Figure S3). The Pearson correlations and slopes were equally good for the IgG and IgM singleplex data versus the multiplex data (see Supporting Information, Figure S3).

Next, we tested the multiplex assay with a more challenging sample. Because IgA signal loss is primarily due to fluorophorefluorophore interactions between the Alexa Fluor 488 dye on the anti-IgA secondary antibody and the Alexa Fluor 546 dye on the anti-IgG secondary antibody, signal loss is most likely to occur in serum samples with very high antibody levels. We have previously profiled serum samples from 220 healthy subjects on our array. ${ }^{16}$ We selected a serum sample (\#F50867-08) that had the highest anti-glycan antibody signals from the cohort, thus placing it in the top $1 \%$ of all serum samples found in healthy subjects. For the secondary antibodies with standard dye loading, the singleplex versus multiplex IgA signals had a correlation $(R)$ of only 0.90 , and 88 array components lost more than $50 \%$ of their original IgA signal (see Supporting Information, Figure S4). As anticipated, IgA signal loss was a major problem for this sample. Fortunately, with the lowerloaded secondary antibodies, the singleplex and multiplex signals had a correlation $(R)$ of 0.98 and only 12 components lost more than $50 \%$ of their original IgA signal. Rhamnose has especially high IgA and IgG signals for most individuals and is one of the glycans that is most prone to IgA signal loss when comparing the singleplex versus multiplex signals. For this serum sample, rhamnose lost $68 \%$ of its signal using the secondary antibodies with standard dye loading but only $3 \%$ with the low dye-loaded secondary antibodies.
Taken together, the results demonstrate that the use of low dye-loaded secondary antibodies will provide substantially improved results and consistent data between singleplex and multiplex assays for all but the most extreme cases. Although fewer dyes per molecule of secondary results in lower signals, we could easily increase the signals by increasing the sensitivity setting on the array scanner. At our standard working serum dilutions of $1: 50$ to $1: 200$, the multiplex assay should provide reliable results for the vast majority of subjects.

Application of the Multiplex Assay for Profiling Antiglycan IgA, IgG, and IgM Repertoires in Humans. While there have been numerous studies profiling serum anti-glycan IgG and/or IgM on glycan microarrays (for some recent examples, see refs; ${ }^{4-16}$ for a recent review, see ref 1$)$, much less is known about serum anti-glycan IgA and the relationships between $\operatorname{IgA}, \operatorname{IgG}$, and $\operatorname{IgM}$ repertoires. In addition, little is known about natural biological variations in anti-glycan IgA repertoires over time and whether variations in IgA are related to variations in IgG and/or IgM. To gain new insight, we used the multiplex assay to profile antibody levels to 500 different glycans and glycoproteins in serum samples taken from seven healthy individuals collected at multiple time points over a 3-month period.

The antibody repertoires for each individual and each isotype are illustrated in Figure 1. All three isotypes recognized similar glycan families. Examples of families with high signals are nonhuman antigens, the blood group $\mathrm{A}$ and $\mathrm{B}$ antigens, Lewis $\mathrm{C}$ and sialylated Lewis C, neutral glycolipids (e.g. GA1, GA2, Gb3, $\mathrm{Gb} 4$, and Gb5), and peptides containing the clustered $\mathrm{Tn}$

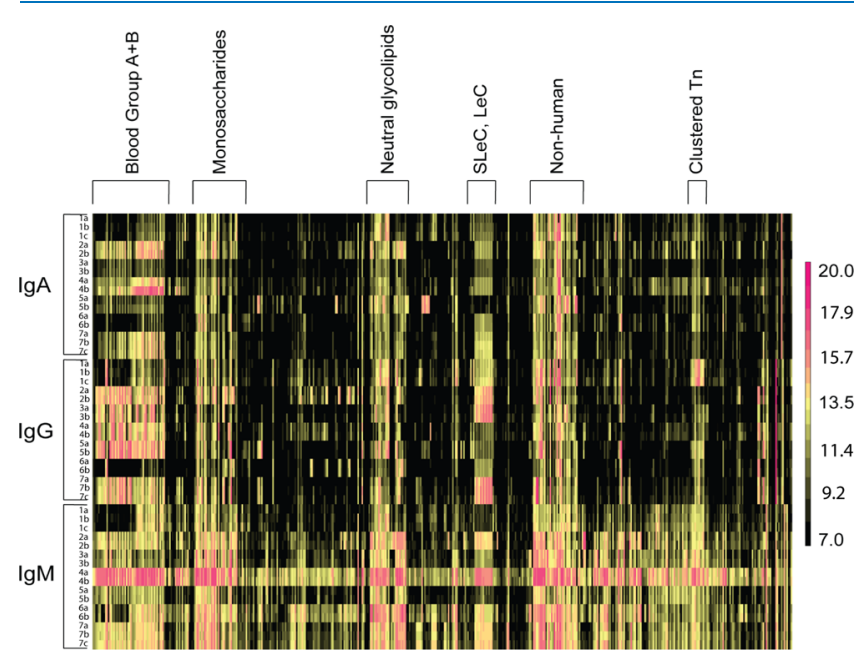

Figure 1. Heat map comparing $\operatorname{IgA} \operatorname{IgG}$, and $\operatorname{IgM}$ antibody profiles. IgA, IgG, and IgM profiles for seven subjects were evaluated on an array with 500 components using the multiplex assay. Data for each subject are shown in rows. Data for each glycan/array component are shown in columns. Signals (raw fluorescence units, RFU) are on $\log 2$ scale. Glycans are organized into families in the columns (several families are highlighted). Black boxes indicate no measurable signal in our assay. Serum samples are labeled with subject number and letter for the time point (e.g. $1 \mathrm{a}, 1 \mathrm{~b}$, and $1 \mathrm{c}$ are the three time points for subject 1 ). Subjects are arranged in the same order for IgA, IgG, and IgM. SLeC is 3 'sialyl Lewis C; LeC is Lewis C; examples of non-human antigens include rhamnose, $\alpha$-Gal, and the Forssman antigen. Clustered Tn antigens are glycopeptides, wherein there are two or more GalNAc- $\alpha$ $\mathrm{Ser} / \mathrm{Thr}$ residues. For a full list of array components and descriptions, see Supporting Information. 
A)

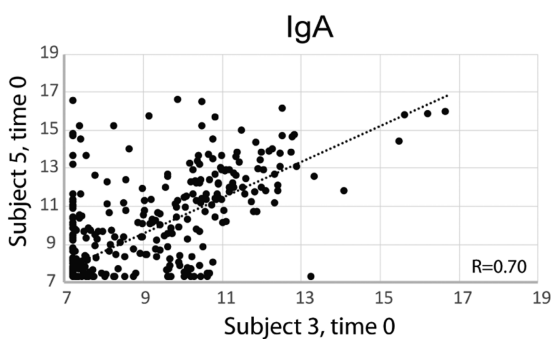

C)

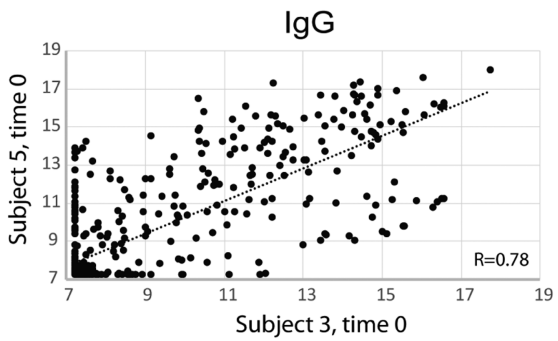

E)

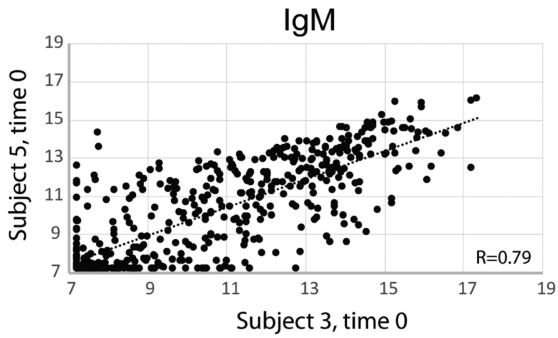

B)

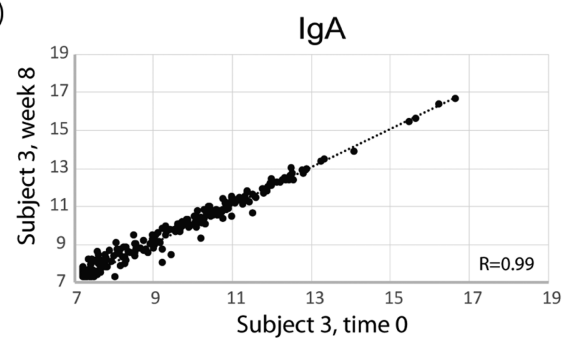

D)

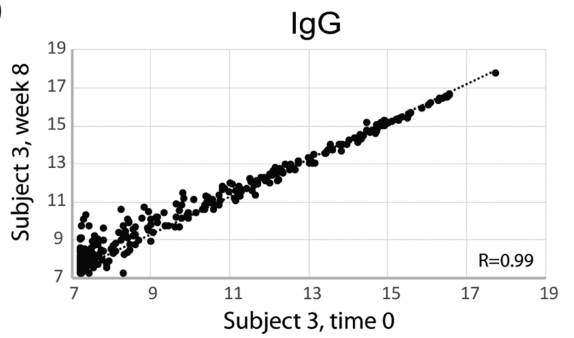

F)

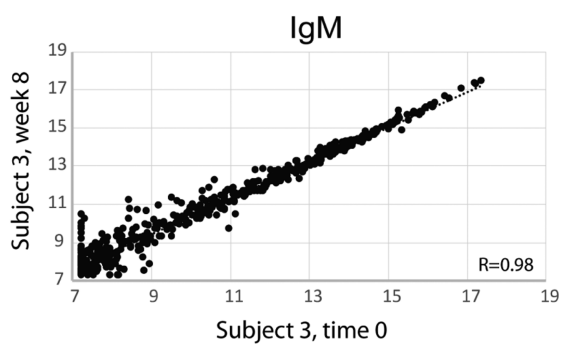

Figure 2. Comparison of antibody signals for the same isotype. For each isotype, correlations were evaluated within the same subject at different time points or from each subject to all other subjects. Representative examples are shown. Signals for each glycan in subject 3 at week 0 were plotted vs subject $5(\mathrm{~A}, \mathrm{C}, \mathrm{E})$ or vs subject 3 at week $8(\mathrm{~B}, \mathrm{D}, \mathrm{F})$. Antibody profiles from the same subject at different time points are very highly correlated $(r=$ 0.98-0.99). Signals (RFU) are on $\log 2$ scale. Isotype profiles from different subjects have a significant correlation.

antigen. IgM antibodies recognized the greatest range of glycans with the greatest signal.

For each isotype, we systematically compared antibody profiles from person to person. A variety of genetic and environmental factors can influence antibody repertoires. For example, age, blood type, and vaccination history can all affect antibody populations in an individual. ${ }^{16,20}$ Relationships were evaluated by plotting signals for a given isotype in one person versus signals for the same isotype in other people (e.g., IgA signals in subject 1 vs IgA signals in subject 2 ; for representative examples, see Figure $2 \mathrm{~A}, \mathrm{C}, \mathrm{E})$. All possible permutations were evaluated, and the median Pearson correlation constants were determined. For IgA, the median Pearson correlation constant from person to person was 0.69. For IgG, the median Pearson correlation constant from person to person was 0.68 , and for IgM it was 0.73 . Therefore, each isotype demonstrated a significant correlation among individuals.

We also evaluated relationships between different isotypes within the same individual and among different individuals. IgA, IgG, and IgM profiles were more consistent with each other within the same individual than in different individuals. The profiles were visualized and quantitated by plotting signals for one isotype versus another isotype (e.g., scatterplot of IgA signals in subject 1 vs IgG signals in subject 1 ; for representative examples, see Figure 3). Again, all possible permutations were evaluated, and the median Pearson correlation constants were determined. When comparing IgA profiles to IgG profiles in the same person, the median Pearson correlation constant was 0.73 (see Table 1). When comparing IgA profiles in one person to IgG profiles in different people, the median Pearson correlation constant decreased to 0.56 . Similar relationships were observed when comparing $\operatorname{IgA}$ to $\operatorname{IgM}$ and $\operatorname{IgG}$ to IgM. For comparisons of IgA profiles to IgM profiles, the median Pearson correlation constant decreased from 0.72 within the same person to 0.62 among different individuals. For IgG versus IgM comparisons, the median Pearson correlation constant decreased from 0.57 to 0.51 .

Variations in Human Anti-Glycan IgA, IgG, and IgM Repertoires over Time. Microarrays are frequently used to identify antibody changes resulting from treatment, infection, or other factors. Antibody levels naturally vary over time and it is important to parse the natural biological fluctuations from the changes directly associated with a clinical treatment. We next evaluated variation over time for our set of subjects.

$\operatorname{IgA}, \operatorname{IgG}$, and $\operatorname{IgM}$ repertoires were all very consistent at different time points within the same person. The consistency can be illustrated by plotting the signals for each glycan on the array at one time point versus another time point for a given subject (for representative examples, see Figure 2B,D,F). Across all the time points, subjects, and isotypes, the median Pearson correlation constant $(R)$ was 0.98 , with the lowest $R$ value being 0.96. For each individual and isotype, we also calculated individual changes in antibody levels over time for each glycan. For 4464 possible changes, only 5.4\% of IgA antibody changes 
Same Subject

A)

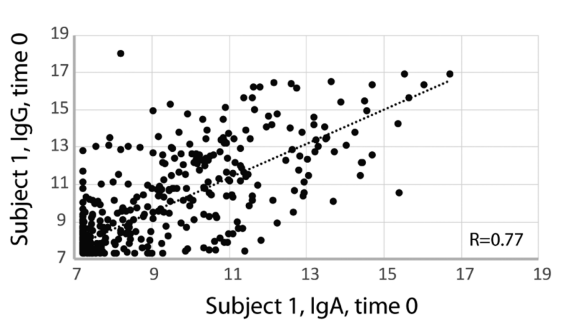

C)

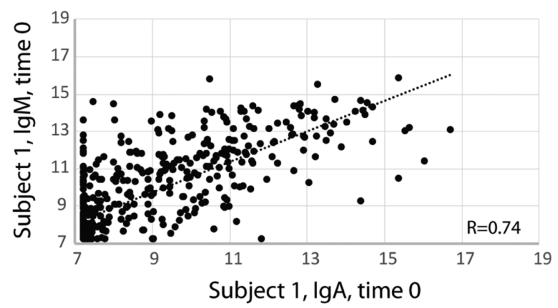

E)

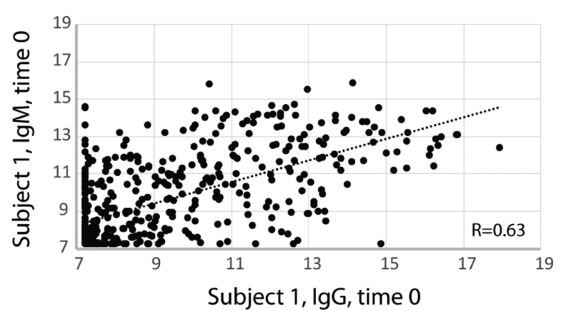

Different Subjects

B)

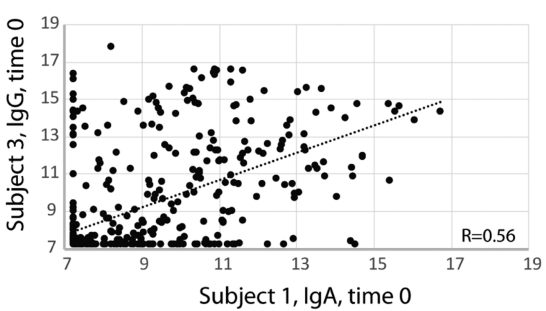

D)

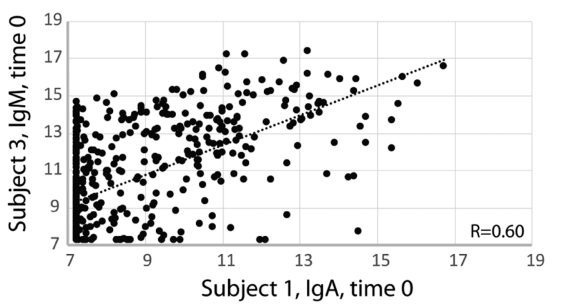

F)

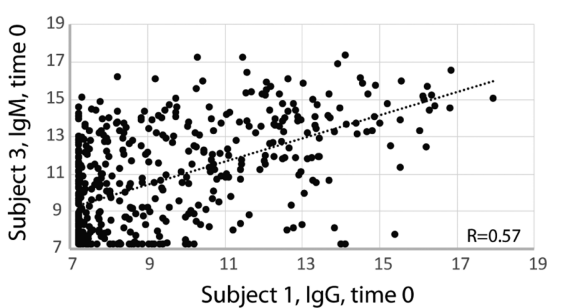

Figure 3. Comparison of antibody signals from one isotype to another. Antibody profiles for each isotype were compared to the other isotypes. Correlations were evaluated within the same subject or across different subjects. Representative examples are shown. For (A,C,E), data for one isotype are compared to a different isotype in the same subject. For (B,D,F), data for one isotype are compared to a different isotype in a different subject. Signals (RFU) are on $\log 2$ scale. Different isotypes are more highly correlated in the same individual than among different individuals.

Table 1. Summary of Pearson Correlation Constants $(R)$ for Comparisons between Various Isotypes

\begin{tabular}{ccccc} 
& & \multicolumn{3}{c}{ isotype 2 } \\
\cline { 3 - 5 } comparison & isotype 1 & IgA & IgG & IgM \\
within the same subject & IgA & $0.99^{a}$ & $0.73^{b}$ & $0.72^{b}$ \\
& IgG & & $0.99^{a}$ & $0.57^{b}$ \\
& IgM & & & $0.99^{a}$ \\
across different subjects & IgA & $0.69^{b}$ & $0.56^{b}$ & $0.62^{b}$ \\
& IgG & & $0.68^{b}$ & $0.51^{b}$ \\
& IgM & & & $0.73^{b}$
\end{tabular}

${ }^{a}$ Compared at different time points from the same subject. ${ }^{b}$ Compared at the same time point.

were 2 -fold or greater; 5.9 and $8.7 \%$ of $\operatorname{IgG}$ and $\operatorname{IgM}$ antibodies had changes greater than 2 -fold as well, respectively. Changes of 4-fold or greater occurred less than $1 \%$ of the time for all isotypes. For reference, 2 -fold or greater changes occur $<3 \%$ of the time and 4 -fold or greater changes occur $<0.006 \%$ of the time due to variability in the assay (based on replicate experiments using the same serum sample). The changes were evenly distributed among the array components, and there were no particular glycan families that consistently demonstrated high variability.

Next, we determined how changes over time for one isotype related to variations in the other isotypes within each individual. Fluctuations from time point to time point within an individual were similar for all three isotypes. For example, IgA levels to most glycans in subject 1 decreased from week 0 to week 7 (90\% of changes were decreases; see Figure 4). Likewise, IgG and IgM levels to most glycans also decreased during this same time period ( $80 \%$ of $\operatorname{IgG}$ changes were decreases; $72 \%$ of IgM changes were decreases in subject 1 ). As a second example, IgA, $\mathrm{IgG}$, and IgM levels to most glycans decreased from week 0 to week 5 in subject 7 ( $82 \%$ of IgA changes, $92 \%$ of IgG changes, and $87 \%$ of IgM changes were decreases). Thus, variations in $\operatorname{IgA}$, IgG, and IgM levels to most glycans appeared to be coordinated.

When assessing variations over multiple time points within a given individual, anti-glycan antibody profiles appeared to fluctuate about a steady state. For example, IgA, IgG, and IgM levels mostly decreased in subject 1 from week 0 to week 7 but then increased from week 7 to week 10 (see Figure 4). The Pearson correlation constants comparing changes from week 0 to 7 versus week 7 to 10 were -0.72 for IgA, -0.66 for IgG, and -0.58 for IgM. These data illustrate the high degree of correlation and the inverse relationship between the changes for the two time frames. The median change from week 0 to week 7 was a $21 \%$ decrease, whereas the median change from week 7 to 10 was a $48 \%$ increase. These changes largely cancelled each other out, as the median change from week 0 to week 10 was a $3 \%$ increase. As another example, IgA, IgG, and IgM mostly decreased in subject 7 from week 0 to week 5 , but they increased from week 5 to week 13. Also, we did not observe any significant new IgA, IgG, or IgM populations during this study, indicating that the set of anti-glycan antibodies present in an individual is stable over weeks to months in healthy adults. 


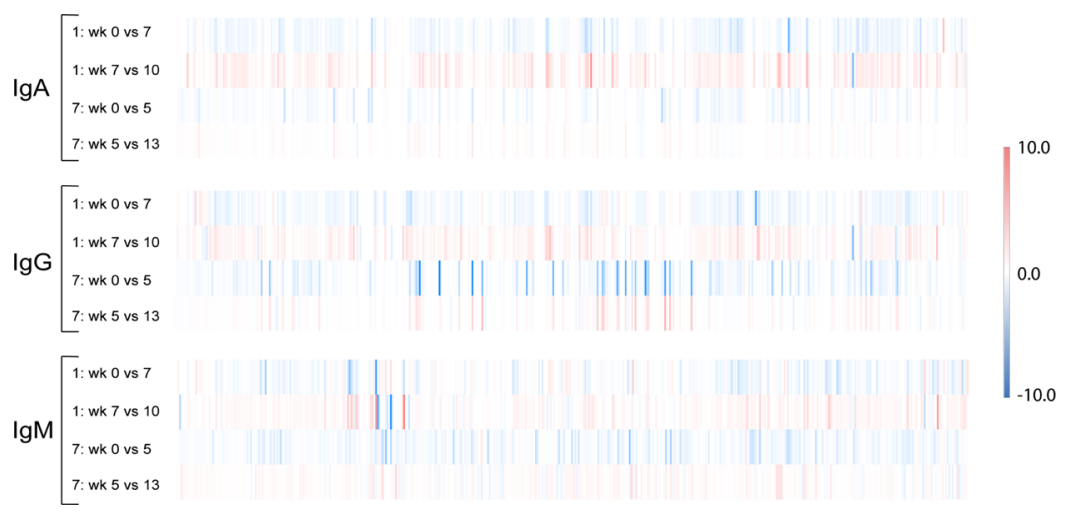

Figure 4. Heat map showing changes for representative subjects. IgA, IgG, and $\operatorname{IgM}$ signals were measured on the glycan microarray at different time points. Changes from one time point to another were determined. Representative data for subjects 1 and 7 are shown in the heat map (data for other subjects can be found in the Supporting Information, Figure S5). White boxes indicate no change; orange boxes indicate an increase; blue boxes indicate decreases. The scale bar and coloring use a linear scale. Rows are labeled with the subject number and the two time points. For example, "1: wk 0 vs 7 " refers to subject 1 , changes from week 0 to week 7 . Changes tend to move in opposite directions at different time points, consistent with a fluctuation about a steady state level. The vast majority of changes shown here are less than 4-fold.
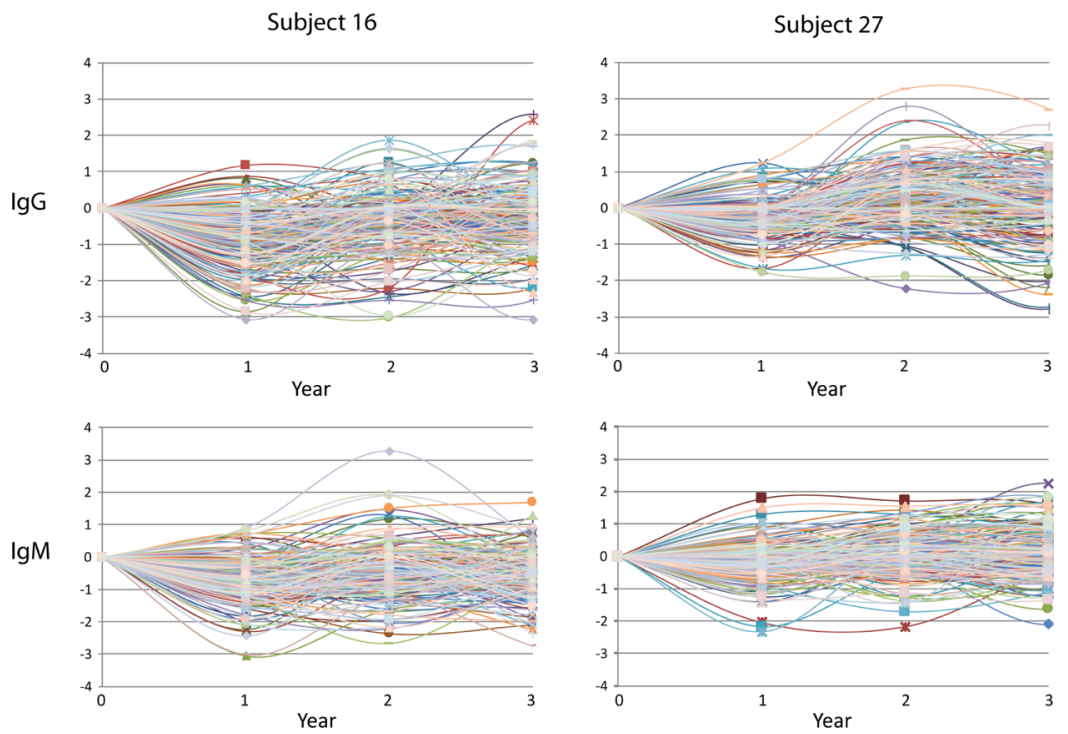

Figure 5. Fluctuations in anti-glycan IgG and IgM over 3 years. IgG and IgM signals were measured on the glycan array at time $0,1,2$, and 3 years. The array had 500 components. Changes at each time point relative to time 0 were determined, and representative examples are shown for two subjects. Signal difference $(y$-axis) is on a $\log 2$ scale. Anti-glycan antibody signals tend to fluctuate about a steady state.

We previously profiled anti-glycan IgG and IgM antibodies present in serum samples from 20 healthy individuals obtained yearly over a 3-year time frame. ${ }^{15}$ To facilitate a better understanding of our new results, we carried out additional analyses on that data. Over this longer timer interval, anti-glycan antibody profiles were very consistent but had a slightly higher level of variability than over weeks to months. For example, IgG and IgM changes of 4-fold or greater occurred about $4-5 \%$ of the time over a 1-3 year time frame. Like the results above, both IgG and IgM repertoires fluctuated about a steady state over a 3year time frame (for representative examples, see Figure 5).

\section{DISCUSSION}

In the first stage of this study, we developed and validated a multiplex assay to simultaneously measure IgA, IgG, and IgM antibodies on a glycan microarray. Antibody binding on the array is detected using fluorophore-labeled secondary antibodies specific for each isotype. For the multiplex assay, we used three different laser/dye pairs to detect different isotypes: a $488 \mathrm{~nm}$ laser/dye pair to detect IgA, a $532 \mathrm{~nm}$ laser/dye pair to detect $\mathrm{IgG}$, and a $635 \mathrm{~nm}$ laser/dye pair to detect IgM. Unfortunately, our initial investigations using commercially available secondary reagents resulted in significant loss of IgA signals in the multiplex relative to the singleplex assay. For example, 54 components lost greater than $50 \%$ of their IgA signal when multiplexed, and 5 components lost greater than $75 \%$ of their IgA signal. If we only consider array components with measurable IgA signals, roughly one-third of that group lost more than $50 \%$ of their signal.

Several lines of evidence indicated that the loss of IgA signal was due to dye-dye interactions (e.g., quenching) between the $488 \mathrm{~nm}$ fluorophore conjugated to the anti-IgA secondary reagent and the $532 \mathrm{~nm}$ fluorophore conjugated to the anti-IgG reagent. First, the $488 \mathrm{~nm}$ fluorophore and $532 \mathrm{~nm}$ fluorophore have some spectral overlap which would allow interaction. Second, loss of IgA signal was largest for array components where IgA and IgG had high signals, situations where dyes are more likely to be in close proximity. Third, when we switched the dyes on the secondary reagents used to detect IgA and IgM, 
we no longer observed loss of IgA signal in the multiplex and we then observed loss of IgM signals.

We evaluated a variety of approaches for addressing the loss of signal. The most fruitful strategy involved decreasing the number of dye molecules attached to each secondary antibody molecule to reduce the number of dyes that are in close enough proximity on the array surface to suffer from dye-dye quenching. Fortunately, this approach led to major improvements. For example, use of low dye-loaded secondary reagents decreased the number of components that lost greater than $50 \%$ of their IgA signal in the multiplex from 54 to just 8 . This approach was validated with multiple serum samples and was found to be effective even for samples with very high anti-glycan antibody levels.

The multiplex assay has several advantages over prior glycan microarray assays for profiling serum antibodies. First, it allows faster acquisition of data while using fewer microarrays and reagents than a single or duplex assay. For example, the longitudinal study carried out in this study required 32 arrays using the multiplex assay, whereas 96 arrays would have been required if each isotype was run individually. Second, the multiplex provides better comparisons among isotypes for a given patient. Because all three isotypes are assayed at the same time using identical experimental conditions and the same array, variability is minimized. Lastly, the multiplex requires less serum for each subject or patient, which can be in very limited supply for many clinical studies.

Although multiplex assays have been reported previously for protein microarrays, our study provides new insights and approaches. In particular, our study highlights signal loss due to fluorophore-fluorophore interactions as a key problem. While prior studies have discussed potential signal loss, the methods and analyses may not have been optimal for detecting this problem. Prior studies have focused on plotting singleplex data versus multiplex data and evaluating Pearson correlation constants. Because fluorophore-fluorophore quenching only affects a subset of signals and there is a large amount of other data, effects on the Pearson correlation constants can be modest. For example, Taghavian et al. state that signal loss was not a problem in their assay based on good Pearson correlation constants; however, the data show a variety of multiplex IgA signals that are lower than expected and the slopes of the lines were lower than $1.0(y=0.84)$, indicating an overall trend of lower than expected signals for the multiplex assay. ${ }^{19}$

We used a combination of approaches to evaluate singleplex versus multiplex data, including determining Pearson correlation constants, evaluating trendline slopes, and counting the number of signals that decrease $50 \%$ or more. In addition, we identified types of serum samples that are especially prone to signal loss, that is, samples with high IgG and IgA signals, where fluorophore-fluorophore interactions are more likely to occur. By using a more sensitive approach for analysis and testing the assay with a "difficult" serum sample, we are better able to detect problems and determine if our assay is suitable. From these insights, we were able to develop a solution for minimizing signal loss: using low dye-loaded secondary reagents. The approaches for detecting signal loss and the strategy for overcoming the problem could be applicable to multiplex assays on protein microarrays as well.

In the second phase of the study, we applied the multiplex assay to study anti-glycan IgA, IgG, and IgM repertoires in healthy human subjects. The study included serum collected at an initial time point as well as serum collected from the same subjects at additional times over several weeks to a few months. Many other studies have reported information about anti-glycan IgG and/or IgM repertoires or combined $\operatorname{IgA} / \operatorname{IgG} / \operatorname{IgM}$ repertoires (for some recent examples, see refs; ${ }^{4-16}$ for a recent review, see ref 1 ). Very few studies have evaluated anti-glycan IgA, and these studies profiled IgA on small arrays, evaluated very few serum samples, and/or did not evaluate any other isotypes. ${ }^{10,11,21-24}$ Consequently, little is known about serum anti-glycan IgA or about relationships between anti-glycan IgA and other isotypes. Additionally, biological variation over time for anti-glycan IgA or whether those variations are related to IgG and/or IgM variations over time have not been evaluated.

Our study provides several key insights. First, in a textbook antibody response, IgM antibodies are formed within a few days after exposure to an antigen. After 1-2 weeks, IgM antibodies decrease and IgG antibodies are produced. The IgM response can be short-lived, whereas IgG responses can be long lasting. From this classical model, one might expect IgM antibody repertoires to be dynamic and variable, while IgG repertoires are more stable. We found that anti-glycan IgA, IgG, and IgM repertoires are all highly stable within an individual over time. When comparing antibody profiles within an individual over weeks to months, Pearson correlation constants were typically 0.99 . Moreover, changes of 4 -fold or greater occurred less than $1 \%$ of the time, and over $1-3$ years changes of 4 -fold or greater occurred less than $5 \%$ of the time, even for IgM. The changes that did occur would be best described as fluctuations about a steady state, rather than changes resulting from a dynamic repertoire, wherein new subpopulations of anti-glycan antibodies continually develop while other subpopulations disappear. Therefore, our results demonstrate that anti-glycan IgA, IgG, and IgM repertoires are stable and tightly regulated within an individual over time. Stable expression of an antibody population throughout life is a common feature of "natural antibodies", or antibodies derived from B1 cells. ${ }^{25}$ Therefore, many of the anti-glycan antibodies that we are detecting, especially the IgM, may be natural antibodies.

The datasets that we report and the information about variability are especially useful for identifying biomarkers and anti-glycan antibody responses that are relevant to biology and medicine. To identify changes induced by vaccination, infection, or disease, one must first understand and define the natural biological variations over time in healthy subjects. Our results provide a baseline for identifying changes induced by treatment or disease that are beyond what one would expect from natural fluctuations over time. In addition, our studies provide insight into how that threshold might change based on the time frame of the study. For a study looking at changes over weeks to months, a threshold of 2- to 3-fold might be sufficient, as changes of this magnitude occur only rarely due to natural variations over this time period. For a study evaluating changes over longer time periods (e.g., years), a threshold of 4-fold or great might be better.

Our study also indicates that anti-glycan IgA repertoires are highly correlated with IgG and IgM repertoires within an individual. At a given time point, the median Pearson correlations between isotypes were 0.73 ( $\operatorname{IgA}$ vs $\operatorname{IgG}), 0.72$ (IgA vs IgM), and 0.57 (IgG vs IgM). The most highly correlated repertoires were IgA and IgG in subject 7, with a Pearson correlation of 0.83 . Correlations were higher within the same individual than among different subjects. Changes in IgA, IgG, and IgM were also correlated. Taken together, these results suggest that the generation of anti-glycan IgA, IgG, and IgM 
repertoires may be coordinated and that expression levels are coregulated within an individual.

Several limitations of our study should be noted. First, our array contains hundreds of glycans and glycopeptides; however, there are numerous other glycans that are not represented. For example, we have little representation of glycosaminoglycans and microbial polysaccharides. Therefore, our observations and conclusions may or may not apply to antibodies that are not detected on our array. Second, the study included a limited number of subjects, and all subjects were healthy. Larger studies and evaluation of individuals with various diseases or other conditions will be needed to further evaluate our findings.

Overall, our study highlights the advantages of combining the multiplex assay with glycan microarray technology for studying and understanding immune recognition of glycans. The ability to simultaneously profile IgA, IgG, and IgM antibodies to approximately 500 glycans and glycopeptides on the array revealed unique insights and systems-level relationships that would be difficult to uncover using traditional approaches and assays. This combination will be especially useful for identifying anti-glycan antibody subpopulations relevant to vaccine development, immunity to pathogens, autoimmunity, allergy, and diseases.

\section{MATERIALS AND METHODS}

Secondary Antibodies. A table of secondary antibodies and dyes evaluated in this study can be found in the Supporting Information (Table S1). The final secondary antibodies used for the multiplex assay were as follows. Alexa Fluor 488 Antibody Labeling Kit (Thermo Fisher, A-20181) was used to label the unconjugated goat anti-human IgA antibody (Jackson Immuno 109.005.011, $1.5 \mathrm{mg} / \mathrm{mL})$. The Alexa Fluor 546 Antibody Labeling Kit (Thermo Fisher, A-20183) was used to label the goat anti-human IgG secondary antibody (Jackson Immuno 109.005.098, $1.5 \mathrm{mg} / \mathrm{mL})$. CF633 succinimidyl ester dye (Sigma-Aldrich; SCJ4600039) was used to label the goat antihuman IgM antibody (Jackson Immuno, 109.005.129). The degree of labeling was determined using the spectral properties of the dye following the manufacturer's instructions. Additional experimental details can be found in the Supporting Information.

Serum Samples. The F58067-08 sample used for testing and multiplex validation was from Valley Biomedical Products and Services (Winchester, VA). The reference serum was pooled from 10 samples purchased from Valley Biomedical Products and Services. Sera used for the longitudinal study were obtained from seven healthy adult male donors through the Research Donor Program at the National Institutes of Health Clinical Center, Department of Transfusion Medicine (for additional information see Supporting Information, Table S2). Samples were tested in accordance with FDA regulations and found to be negative for $\mathrm{HIV} 1 / 2 \mathrm{AB}, \mathrm{HCV} \mathrm{AB}$, and nonreactive for HBSAG, HIV-1 RNA, HCV RNA, and STS. All samples were obtained in serum separator tubes, processed within $4 \mathrm{~h}$ of blood collection, and stored at $-80{ }^{\circ} \mathrm{C}$ until assayed. All samples had been thawed and refrozen at least once but not more than 3 times. In prior work, we have found that up to 3 freeze-thaw cycles have no effect on anti-glycan antibody profiles. ${ }^{26}$

Glycan Microarray Fabrication. Glycans and glycopeptides used for microarrays were conjugated to either bovine serum albumin (BSA) or human serum albumin (HSA) to produce neoglycoproteins that approximate the density of spacing of natural glycoproteins. ${ }^{27}$ Some glycans were conjugated at high and low densities, as denoted by the number following the array component name. The array platform, printing, and assay have been described previously, ${ }^{28}$ along with analysis of reproducibility ${ }^{20}$ and validation with numerous antibodies and lectins. ${ }^{29-33}$ Array printing was done on a MicroGrid II arrayer (Biorobotics) by SMP2 pins (Arrayit). Neoglycoproteins were printed at $125 \mu \mathrm{g} / \mathrm{mL}$ and glycoproteins were printed at $200 \mu \mathrm{g} / \mathrm{mL}$ in duplicate on epoxy-coated slides (SME2, Arrayit); for a full list of the 500 array components, see Supporting Information. Atto555 azide dye (Thermo Scientific) at $0.7 \mu \mathrm{g} / \mathrm{mL}$ was mixed in the print buffer to evaluate print quality prior to performing experimental assays. The soluble dye is washed away before each experiment. Each slide contained 16 identical wells with an average spot size of $80 \mu \mathrm{m}$. Representative slides from each print batch were assessed for quality and reproducibility using a standard set of lectins [ConA (Manbinding lectin) and WGA (GlcNAc-binding lectin) from Vector Laboratories; HPA (GalNAc-binding lectin) from SigmaAldrich] and monoclonal antibodies [CLCP-19B (blood group B-binding antibody; BioLegend) and T36 (blood group A-binding antibody; Genetex)]. The slides were stored in vacuum-sealed boxes at $-20{ }^{\circ} \mathrm{C}$ until use.

High-Throughput Profiling of Serum Anti-glycan IgA, IgG, and IgM Antibodies. Prior to each experiment, each microarray slide was scanned in an InnoScan $1100 \mathrm{AL}$ fluorescence scanner to check for any defects and missing print spots. The slides were placed in 16-well modules using metal clips (Grace Bio-Labs) to separate the 16 identical arrays and allow for different experimental conditions. The array slides were blocked overnight at $4{ }^{\circ} \mathrm{C}$ in $3 \%$ BSA, phosphate buffered saline (PBS) $(200 \mu \mathrm{L} /$ well $)$ and then washed six times with PBS buffer ( $\mathrm{pH} 7.4$ ) containing $0.05 \%$ Tween-20 (PBST). Human sera were diluted $1: 25$ or $1: 50$ in $3 \%$ BSA and $1 \%$ HSA in PBST and added to assigned wells on the slide (100 $\mu \mathrm{L} /$ well $)$. All serum samples were measured in duplicate wells printed by different pins in order to minimize pin and slide variations. All samples from a given subject were assayed on the same day to minimize day-to-day assay variation. To help detect potential experimental variability, we include a variety of controls on the array, such as printed human IgA, IgG, and IgM spots, unmodified BSA and HSA, and printed dyes. After incubating for $4 \mathrm{~h}$ at $37^{\circ} \mathrm{C}$ with gentle agitation $(100 \mathrm{rpm})$, the slides were washed six times in PBST $(200 \mu \mathrm{L} /$ well $)$. To detect bound serum antibodies, secondary antibodies were diluted 1:500 in $3 \%$ HSA and $1 \%$ BSA PBS and added to the slide $(100 \mu \mathrm{L} /$ well $)$. Slides were covered with aluminum foil to prevent photobleaching and then incubated for $2 \mathrm{~h}$ at $37{ }^{\circ} \mathrm{C}$ with gentle agitation. Following six washes with PBST, slides were removed from the module and soaked in PBST for 5 min prior to being dried in a centrifuge at $1000 \mathrm{rpm}$ for $5 \mathrm{~min}$.

Image Analysis and Data Processing. The arrays were scanned at $5 \mu \mathrm{m}$ pixel resolution with an InnoScan $1100 \mathrm{AL}$ fluorescence scanner. The photomultiplier tube (PMT) settings were the same for all experiments to limit unintentional signal variation. Slides were scanned at "high" and "low" PMT settings (for the $488 \mathrm{~nm}$ laser, high pmt $=100$ and low pmt $=55$; for the $532 \mathrm{~nm}$ laser, high pmt $=20$ and low $=5$; for the $635 \mathrm{~nm}$ laser, high $=9$ and low = 4) to increase the dynamic range and appropriately scale-saturated components. Spots were defined as circular features with diameter $60-80 \mu \mathrm{m}$, and fluorescence intensity for each component was quantified with GenePix 7.0 software (Molecular Probes). The GenePix array file was manually adjusted for each spot to reflect their actual sizes and 
placements. Any features marked as missing or defective in the prescan were excluded from further analysis. The local background corrected median was used for data analysis, and spots with intensity lower than $150 \mathrm{RFU}$ ( $1 / 2$ the typical IgM background) were set to 150 . The signals for replicate spots on duplicate wells were averaged and log-transformed (base 2) for future analysis. Representative images of array data (prescans and post-assay scans) can be found in the Supporting Information (see Figure S1).

\section{ASSOCIATED CONTENT}

\section{S Supporting Information}

The Supporting Information is available free of charge on the ACS Publications website at DOI: 10.1021/acsomega.8b02238.

Supplementary materials and methods; secondary antibody + fluorophore data; summary of subjects and samples used in the longitudinal study; pre- and postexperiment images; scatter plot of IgA signals; comparison of singleplex and multiplex signals for $\operatorname{IgA}, \operatorname{IgG}$, and IgM after optimization; loss of IgA signal observed for a high IgG; and heat map showing changes for subjects 2-6 (PDF)

Experiment summary and full glycan microarray data (XLSX)

\section{AUTHOR INFORMATION}

\section{Corresponding Author}

*E-mail: gildersj@mail.nih.gov.

ORCID

Jeffrey C. Gildersleeve: 0000-0002-3744-6423

Notes

The authors declare no competing financial interest.

\section{ACKNOWLEDGMENTS}

We thank the Consortium for Functional Glycomics (GM62116; The Scripps Research Institute), Professor Tom Tolbert (University of Kansas), Professor Lai-Xi Wang (University of Maryland), Professor Xuefei Huang (Michigan State University), Professor Todd Lowary (University of Alberta), Dr. Joseph Barchi (National Cancer Institute), and Omicron Biochemicals Inc. for contributing glycans for the array. This work was supported by the Intramural Research Program of the National Cancer Institute, NIH.

\section{REFERENCES}

(1) Muthana, S. M.; Gildersleeve, J. C. Glycan Microarrays: Powerful Tools for Biomarker Discovery. Cancer Biomarkers 2014, 14, 29-41.

(2) Amon, R.; Reuven, E. M.; Leviatan Ben-Arye, S.; Padler-Karavani, V. Glycans in immune recognition and response. Carbohydr. Res. 2014, $389,115-122$.

(3) Rabinovich, G. A.; van Kooyk, Y.; Cobb, B. A. Glycobiology of immune responses. Ann. N.Y. Acad. Sci. 2012, 1253, 1-15.

(4) Burford, B.; Gentry-Maharaj, A.; Graham, R.; Allen, D.; Pedersen, J. W.; Nudelman, A. S.; Blixt, O.; Fourkala, E. O.; Bueti, D.; Dawnay, A.; Ford, J.; Desai, R.; David, L.; Trinder, P.; Acres, B.; Schwientek, T.; Gammerman, A.; Reis, C. A.; Silva, L.; Osório, H.; Hallett, R.; Wandall, H. H.; Mandel, U.; Hollingsworth, M. A.; Jacobs, I.; Fentiman, I.; Clausen, H.; Taylor-Papadimitriou, J.; Menon, U.; Burchell, J. M. Autoantibodies to MUC1 glycopeptides cannot be used as a screening assay for early detection of breast, ovarian, lung or pancreatic cancer. $B$. J. Cancer 2013, 108, 2045-2055.

(5) Pochechueva, T.; Alam, S.; Schötzau, A.; Chinarev, A.; Bovin, N. V.; Hacker, N. F.; Jacob, F.; Heinzelmann-Schwarz, V. Naturally occurring anti-glycan antibodies binding to Globo $\mathrm{H}$-expressing cells identify ovarian cancer patients. J. Ovarian Res. 2017, 10, 8.

(6) Schneider, C.; Smith, D. F.; Cummings, R. D.; Boligan, K. F.; Hamilton, R. G.; Bochner, B. S.; Miescher, S.; Simon, H.-U.; Pashov, A.; Vassilev, T.; von Gunten, S. The human IgG anti-carbohydrate repertoire exhibits a universal architecture and contains specificity for microbial attachment sites. Sci. Transl. Med. 2015, 7, 269 ra1.

(7) Mickum, M. L.; Prasanphanich, N. S.; Song, X.; Dorabawila, N.; Mandalasi, M.; Lasanajak, Y.; Luyai, A.; Secor, W.E.; Wilkins, P. P.; Van Die, I.; Smith, D. F.; Nyame, A. K.; Cummings, R. D.; Rivera-Marrero, C. A. Identification of antigenic glycans from Schistosoma mansoni by using a shotgun egg glycan microarray. Infect. Immun. 2016, 84, 13711386.

(8) van Diepen, A.; van der Plas, A.-J.; Kozak, R. P.; Royle, L.; Dunne, D. W.; Hokke, C. H. Development of a Schistosoma mansoni shotgun $\mathrm{O}$-glycan microarray and application to the discovery of new antigenic schistosome glycan motifs. Int. J. Parasitol. 2015, 45, 465-475.

(9) Jacob, F.; Anugraham, M.; Pochechueva, T.; Tse, B. W. C.; Alam, S.; Guertler, R.; Bovin, N. V.; Fedier, A.; Hacker, N. F.; Huflejt, M. E.; Packer, N.; Heinzelmann-Schwarz, V. A. The glycosphingolipid P1 is an ovarian cancer-associated carbohydrate antigen involved in migration. Br. J. Cancer 2014, 111, 1634-1645.

(10) Broecker, F.; Martin, C. E.; Wegner, E.; Mattner, J.; Baek, J. Y.; Pereira, C. L.; Anish, C.; Seeberger, P. H. Synthetic Lipoteichoic Acid Glycans Are Potential Vaccine Candidates to Protect from Clostridium difficile Infections. Cell Chem. Biol. 2016, 23, 1014-1022.

(11) Jeyakanthan, M.; Meloncelli, P. J.; Zou, L.; Lowary, T. L.; Larsen, I.; Maier, S.; Tao, K.; Rusch, J.; Chinnock, R.; Shaw, N.; Burch, M.; Beddows, K.; Addonizio, L.; Zuckerman, W.; Pahl, E.; Rutledge, J.; Kanter, K. R.; Cairo, C. W.; Buriak, J. M.; Ross, D.; Rebeyka, I.; West, L. J. ABH-Glycan Microarray Characterizes ABO Subtype Antibodies: Fine Specificity of Immune Tolerance after ABO-Incompatible Transplantation. Am. J. Transplant. 2016, 16, 1548-1558.

(12) Campbell, C. T.; Gulley, J. L.; Oyelaran, O.; Hodge, J. W.; Schlom, J.; Gildersleeve, J. C. Serum Antibodies to Blood Group A Predict Survival on PROSTVAC-VF. Clin. Cancer Res. 2013, 19, 12901299.

(13) Campbell, C. T.; Gulley, J. L.; Oyelaran, O.; Hodge, J. W.; Schlom, J.; Gildersleeve, J. C. Humoral response to a viral glycan correlates with survival on PROSTVAC-VF. Proc. Natl. Acad. Sci. U.S.A. 2014, 111, E1749-E1758.

(14) Xia, L.; Schrump, D. S.; Gildersleeve, J. C. Whole-Cell Cancer Vaccines Induce Large Antibody Responses to Carbohydrates and Glycoproteins. Cell Chem. Biol. 2016, 23, 1515-1525.

(15) Scheepers, C.; Chowdhury, S.; Wright, W. S.; Campbell, C. T.; Garrett, N. J.; Abdool Karim, Q.; Abdool Karim, S. S.; Moore, P. L.; Gildersleeve, J. C.; Morris, L. Serum glycan-binding IgG antibodies in HIV-1 infection and during the development of broadly neutralizing responses. AIDS 2017, 31, 2199-2209.

(16) Muthana, S. M.; Gildersleeve, J. C. Factors Affecting Anti-Glycan IgG and IgM Repertoires in Human Serum. Sci. Rep. 2016, 6, 19509.

(17) Renault, N. K.; Gaddipati, S. R.; Wulfert, F.; Falcone, F. H.; Mirotti, L.; Tighe, P. J.; Wright, V.; Alcocer, M. J. C. Multiple protein extract microarray for profiling human food-specific immunoglobulins A, M, G and E. J. Immunol. Meth. 2011, 364, 21-32.

(18) Zhang, B.; Kumar, R. B.; Dai, H.; Feldman, B. J. A plasmonic chip for biomarker discovery and diagnosis of type 1 diabetes. Nat. Med. 2014, 20, 948-953.

(19) Taghavian, O.; Jain, A.; Joyner, C. J.; Ketchum, S.; Nakajima, R.; Jasinskas, A.; Liang, L.; Fong, R.; King, C.; Greenhouse, B.; Murphy, M.; Bailey, J.; Galinski, M. R.; Barnwell, J. W.; Plowe, C. V.; Davies, D. H.; Felgner, P. L. Antibody Profiling by Proteome Microarray with Multiplex Isotype Detection Reveals Overlap between Human and Aotus nancymaae Controlled Malaria Infections. Proteomics 2018, 18, 1700277.

(20) Oyelaran, O.; McShane, L. M.; Dodd, L.; Gildersleeve, J. C. Profiling human serum antibodies with a carbohydrate antigen microarray. J. Proteome Res. 2009, 8, 4301-4310. 
(21) Muthana, S. M.; Xia, L.; Campbell, C. T.; Zhang, Y.; Gildersleeve, J. C. Competition Between Serum IgG, IgM, and IgA Anti-Glycan Antibodies. PLoS One 2015, 10, No. e0119298.

(22) Oberli, M. A.; Hecht, M.-L.; Bindschädler, P.; Adibekian, A.; Adam, T.; Seeberger, P. H. A Possible Oligosaccharide-Conjugate Vaccine Candidate for Clostridium difficile Is Antigenic and Immunogenic. Chem. Biol. 2011, 18, 580-588.

(23) Martin, C. E.; Broecker, F.; Oberli, M. A.; Komor, J.; Mattner, J.; Anish, C.; Seeberger, P. H. Immunological evaluation of a synthetic clostridium difficile oligosaccharide conjugate vaccine candidate and identification of a minimal epitope. J. Am. Chem. Soc. 2013, 135, 97139722.

(24) Padler-Karavani, V.; Tremoulet, A. H.; Yu, H.; Chen, X.; Burns, J. C.; Varki, A. A Simple Method for Assessment of Human Anti-Neu5Gc Antibodies Applied to Kawasaki Disease. PLoS One 2013, 8, No. e58443.

(25) Panda, S.; Ding, J. L. Natural antibodies bridge innate and adaptive immunity. J. Immunol. 2015, 194, 13-20.

(26) Muthana, S. M.; Gulley, J. L.; Hodge, J. W.; Schlom, J.; Gildersleeve, J. C. ABO Blood Type Correlates with Survival on Prostate Cancer Vaccine Therapy. Oncotarget 2015, 6, 32244-32256.

(27) Zhang, Y.; Gildersleeve, J. C. General Procedure for the Synthesis of Neoglycoproteins and Immobilization on Epoxide-Modified Glass Slides. In Carbohydrate Microarrays: Methods and Protocols; Chevolot, Y., Ed.; Humana Press, 2012; Vol. 808, pp 155-165.

(28) Campbell, C. T.; Zhang, Y.; Gildersleeve, J. C. Construction and Use of Glycan Microarrays. Curr. Protoc. Chem. Biol. 2010, 2, 37-53.

(29) Lubkowski, J.; Durbin, S. V.; Silva, M. C. C.; Farnsworth, D.; Gildersleeve, J. C.; Oliva, M. L. V.; Wlodawer, A. Structural analysis and unique molecular recognition properties of a Bauhinia forficata lectin that inhibits cancer cell growth. FEBS J. 2017, 284, 429-450.

(30) Gibadulin, R.; Farnsworth, D. W.; Barchi, J. J.; Gildersleeve, J. C. GalNAc-Tyrosine Is a Ligand of Plant Lectins, Antibodies, and Human and Murine Macrophage Galactose-Type Lectins. ACS Chem. Biol. 2017, 12, 2172-2182.

(31) Gildersleeve, J. C.; Wright, W. S. Diverse molecular recognition properties of blood group A binding monoclonal antibodies. Glycobiology 2016, 26, 443-448.

(32) Li, Q.; Anver, M. R.; Li, Z.; Butcher, D. O.; Gildersleeve, J. C. GalNAc $\alpha 1$-3Gal, a New Prognostic Marker for Cervical Cancer. Int. J. Cancer 2010, 126, 459-468.

(33) Manimala, J. C.; Roach, T. A.; Li, Z.; Gildersleeve, J. C. Highthroughput carbohydrate microarray profiling of 27 antibodies demonstrates widespread specificity problems. Glycobiology 2007, 17, $17 \mathrm{C}-23 \mathrm{C}$. 